\title{
Tratamiento de la discapacidad en el marco auto-normativo de los medios de comunicación españoles
}

\author{
Yordhana GómEz \\ Universidad de Alicante \\ yordhanagomez@gmail.com \\ Marta Martín LlaGunO \\ Universidad de Alicante \\ marta.martin@us.es \\ Andreu Castellet \\ Universidad de Murcia
}

\section{Resumen:}

Los modelos de inserción social de la discapacidad han sufrido una evolución impulsada por la Convención Internacional sobre los Derechos de las Personas con Discapacidad. Partiendo de los conceptos expuestos en dicha Convención, este trabajo explora el tratamiento específico de estas cuestiones en los medios de comunicación españoles. Para ello realiza un análisis de contenido en 5 libros de estilo de diarios y agencias de noticias.

Palabras Clave: Discapacidad; medios de comunicación; prensa; libro de estilo; manual de estilo

\section{Treatment of disability in the self-regulatory framework of the Spanish media}

\begin{abstract}
:
Social integration models of disability have undergone an evolution reinforced by the International Convention on the Rights of Persons with Disabilities. In accordance with the concepts of disability and accessibility defined by the Convention, this research aims to find out if the Spanish media offer due, specific treatment to these issues. To achieve our research goal, we undergo a content analysis of the stylebooks of 5 major Spanish media -newspapers and news agencies.
\end{abstract}

Key Words: Disability; mass media; news media; style manual; style guide

\section{Referencia normalizada:}

Gómez, Y., Martín Llaguno, M. y Castellet, A. (2014): Tratamiento de la discapacidad en el marco auto-normativo de los medios de comunicación españoles. Historia y Comunicación Social. Vol. 19. Núm. Especial Enero. Págs. 667-679.

Sumario:_1. Estado de la cuestión, 2. Metodología, 3. Resultados, 4. Discusión, 5. Conclusiones, 6. Referencias Bibliográficas, 7.Notas. 


\section{Estado de la cuestión}

La ratificación de la Convención sobre los Derechos de las Personas con Discapacidad (en adelante la Convención) modifica la aproximación pública a este tema. A partir de este hito, se progresa del modelo médico al modelo social, consolidándose un cambio que había comenzado en las décadas anteriores (Filkenstein, 1980; Stibbe, 2004). Este documento desplaza el tratamiento del individuo a las políticas públicas y a la sociedad en su conjunto, reconociendo que la discapacidad es un concepto que evoluciona y que resulta de la interacción entre las personas con deficiencias y las barreras debidas a la actitud y al entorno que evitan su participación plena y efectiva en la sociedad, en igualdad de condiciones con las demás (ONU, 2007: 1). No es de extrañar la referencia a la adopción de medidas pertinentes para asegurar el acceso de las personas con discapacidad, en igualdad de condiciones con las demás, al entorno físico, el transporte, la información y las comunicaciones, incluidos los sistemas y las tecnologías de la información y las comunicaciones (art. 9). Posteriormente, el artículo 21 obliga a los firmantes a adoptar todas las medidas pertinentes para que las personas con discapacidad puedan ejercer el derecho a la libertad de expresión y opinión, incluida la libertad de recabar, recibir y facilitar información e ideas en igualdad de condiciones con las demás y mediante cualquier forma de comunicación. No obstante, la Convención no sólo se ocupa de lo que atañe a los derechos de acceso a los contenidos de los medios de comunicación y de entretenimiento. También impulsa la construcción de la imagen social de este concepto en su artículo 8 que alienta a todos los órganos de los medios de comunicación a que difundan una imagen de las personas con discapacidad que sea compatible con el propósito de la presente Convención (Ibid:10).

Para alcanzar este objetivo es necesario, además de generar nuevas formas de comunicación, modificar y adaptar las existentes porque, a pesar del cambio o la diferencia, las personas intentamos mantener nuestras costumbres y las de los que nos rodean.

Este trabajo pretende ser una aportación al campo multidisciplinar y plurifocal de la discapacidad y los medios (Auslander y Gold, 1999). Como asunto social sobre el que estos últimos pueden jugar un papel relevante (Fernández-Cid, 2010), las definiciones y el tratamiento estilístico que la prensa, la radio y la televisión hagan de la discapacidad contribuirán a la construcción pública de la misma. Por esta razón es necesario revisar los manuales de estilo, que prescriben a los profesionales las fórmulas para tratar distintos supuestos. Pérez Tornero describe estos manuales como repertorios retóricos y éticos, un dispositivo metalingüístico orientado a fijar selecciones léxicas y sintácticas, además de operativas y discursivas (Alcoba, 2009: 202). Por otra parte, Alcoba señala:

Si nos referimos al aspecto de la lengua, podemos decir que un LdE [Libro de Estilo] no es una gramática, ni un diccionario, ni una ortografía; pero el LdE establece, recuerda o precisa algunas reglas gramaticales de la lengua; resuelve cuestiones de regularidad, extensión y uso de algunas palabras; y fija aspectos de sonido o 
de grafía (en las publicaciones escritas), como el uso de la caja (mayúscula o minúscula) o el tipo (tamaño) y características de la letra empleada (redonda, cursiva, etc.). El LdE reúne un conjunto de decisiones sobre aspectos de manifestación variable y estilística de la lengua referidas a los autores y a los textos de un medio de comunicación determinado para su fijación y unificación expresiva. Tiene como objetivo que todos los autores que intervienen en un medio de comunicación adopten la misma solución ante distintas alternativas expresivas o estilísticas (2009: 4-5).

A menudo los libros de estilo ordenan o recomiendan decisiones sobre cuestiones semánticas en las que existen diferentes opciones posibles. Es en estos capítulos donde se tienen en cuenta los matices semióticos de estas decisiones, con lo que se convierten en herramienta de identidad e ideología.

En consecuencia el objetivo de los libros de estilo es aunar las soluciones de los profesionales de un medio ante una situación con múltiples alternativas. Al tratarse de un unificador de identidad, concreta el contrato enunciativo y comunicativo entre el emisor y el receptor. Son por lo tanto estos hechos los que transforman a los libros de estilo en una fuente de información importante sobre la construcción conceptual de una cuestión social.

En el caso de la discapacidad, nuestro interés es averiguar si existe un interés específico por el tema en los libros de estilo, cuál es el enfoque que cada medio da a esta cuestión y si existe un patrón colectivo. Se trata de observar también si la evolución en el tratamiento del tema coincide, o no, con las tendencias marcadas por los organismos internacionales.

\section{Metodología}

Con los precedentes expuestos, planteamos la compilación y revisión de las referencias a la discapacidad y a la accesibilidad en los principales manuales de estilo de los medios de comunicación españoles. Una vez recogidas estas alusiones, nos proponemos identificarlas y valorarlas a la luz de los preceptos y valores contenidos en la Convención sobre Derechos de las Personas con Discapacidad.

Para hacer frente a estos objetivos se ha realizado un análisis de contenido (Piñuel Raigada, 2002) de los principales libros de estilo de los medios de comunicación en España. La muestra ha sido seleccionada de acuerdo a los criterios de audiencia y relevancia (adecuación y reputación). Basándonos en los datos del EGM (Estudio General de Medios, AIMC, 2013) se han escogido los manuales de El País, El Mundo y RTVE. A estos, reforzados por el segundo criterio, se han añadido los de las agencias de noticias EFE (2 libros) y Servimedia. De estos 6 libros de estilo, 4 son formato digital y 2 en papel (EFE).

Se han utilizado para las búsquedas términos clave asociados a los conceptos accesibilidad y discapacidad teniendo en cuenta las evoluciones semánticas del campo, a saber: accesibilidad, accesible, acceso, deficiencia, discapacidad, discapacidad 
visual, diversidad funcional y minusvalía. En un primer momento se ha realizado una localización sistemática de los términos y la recolección del contexto en el que se inscriben con la ayuda del MSWord (2007) y Adobe Acrobat (9.5), en los formatos digitales. En el soporte físico se ha inspeccionado el índice y el diccionario (normalmente apodado "Sobre Léxico").

En una segunda parte, primero se ha realizado un análisis de frecuencias de aparición. Después se han categorizado en un proceso cualitativo según muestra la tabla 1 en el que se ha examinado el contexto proporcionando el análisis de valoraciones y actitudes a través de unidades léxicas y temáticas. Algunos de estos indicadores han sido valorados a través del contexto en el que se inserta el término analizado.

Tabla 1. Ficha de codificación del análisis de contenido de los libros de estilo

\begin{tabular}{|l|l|}
\hline Indicador & Codificación \\
\hline \multirow{2}{*}{ Área } & Discapacidad/General \\
\hline Valor morfosintáctico & Modelos: Médico/Social/ Ninguno \\
\hline \multirow{2}{*}{ Campo semántico } & Estigmatización: SiNo $\mathrm{No}$ \\
\hline \multirow{2}{*}{ Acción afirmativa } & "Palabra a la que va asociado" \\
\hline Valor semántico & SiNo \\
\hline Omisión & $\begin{array}{l}\text { Casos ejemplares/Inclusión/Tiempo específico en antena } \\
\text { Desambiguación del significado }\end{array}$ \\
\hline Especificidad & SiNo \\
\hline
\end{tabular}

Fuente: Elaboración propia

\section{Resultados}

Se han encontrado 171 ocurrencias distribuidas según muestran las tablas 2 y 3 . Servimedia presenta la mayoría de casos (con un total de 94), seguido de RTVE con 52, El País con 17 y El Mundo con 8 apariciones. Aunque la frecuencia general de términos resulta escasa llaman la atención las 36 repeticiones de la palabra acceso en RTVE y las 70 de la palabra discapacidad en Servimedia. El análisis frecuencial muestra una omisión importante de las palabras accesibilidad, discapacidad visual y diversidad funcional. 
Tabla 2. Frecuencia de aparición de las palabras dentro del campo semántico de la accesibilidad

\begin{tabular}{|c|l|c|c|c|}
\hline \multicolumn{2}{|c|}{ Términos } & Accesibilidad & Accesible & Acceso \\
\hline \multirow{3}{*}{ Medio } & Servimedia & 1 & 3 & 8 \\
\cline { 2 - 5 } & El Mundo & 0 & 4 & 3 \\
\cline { 2 - 5 } & El País & 0 & 4 & 4 \\
\cline { 2 - 5 } & RTVE & 1 & 3 & 36 \\
\hline
\end{tabular}

Fuente: Elaboración propia

Tabla 3. Frecuencia de aparición de las palabras dentro del campo semántico de la discapacidad

\begin{tabular}{|c|l|c|c|c|c|c|}
\hline \multicolumn{2}{|c|}{ Términos } & Deficiencia & Discapacidad & $\begin{array}{c}\text { Discapacidad } \\
\text { visual }\end{array}$ & $\begin{array}{c}\text { Diversidad } \\
\text { funcional }\end{array}$ & Minusvalía \\
\hline \multirow{3}{*}{ Medio } & Servimedia & 1 & 70 & 7 & 2 & 2 \\
\cline { 2 - 7 } & El Mundo & 1 & 0 & 0 & 0 & 0 \\
\cline { 2 - 7 } & El País & 3 & 3 & 0 & 0 & 3 \\
\cline { 2 - 7 } & RTVE & 4 & 8 & 0 & 0 & 0 \\
\hline
\end{tabular}

Fuente: Elaboración propia

La tabla 4 muestra los resultados de la ficha de codificación, así como los totales parciales. Al contextualizar los términos, en su mayoría estos poseen una definición general, es decir, no asociada al área de la discapacidad. Sin embargo se puede destacar que en el libro de estilo de El Mundo se utiliza el concepto de accesible al señalar "la importancia de hacer la información comprensible" (El Mundo, 2002:14). Esta dimensión es 1 de los 4 pilares en los estudios de accesibilidad (Ribera, 2009) (los otros 3 son la perceptibilidad ${ }^{1}$, operabilidad ${ }^{2}$ y la robuste ${ }^{3}$ ) entenderemos en consecuencia que este medio le da un valor semántico técnico.

Por su parte, el libro de estilo de El País define la minusvalía como la "situación desventajosa para el individuo" (El País, 2002: 279), a modo de término-paraguas bajo el que se asocian diferenciadamente discapacidad y deficiencia. Esta última es "la pérdida de una estructura o función" (Ibid: 187) que tiene como consecuencia la discapacidad como la "restricción o ausencia de capacidad de realizar una actividad" (Ibid:192) y genera la minusvalía, que limita o impide el normal desenvolvimiento. Todos estos conceptos acaban relacionados con "el margen normal de actuación del ser humano" (Ibid: 279). Al ser definiciones, aparecen como nombres en el texto. Además, en este manual se omite la mención de una discapacidad específica.

En este mismo documento los términos vinculados con el acceso se vinculan con la economía (Ibid: 134) y también existe una referencia clara a la comprensión unida a la "accesibilidad de los contenidos" (Ibid: 18). 
Tabla 4. Resultados según ficha de codificación (detallada en tabla 1)

\begin{tabular}{|c|c|c|c|c|c|c|}
\hline \multirow[b]{2}{*}{ Indicador } & \multirow[b]{2}{*}{ Codificación } & \multicolumn{4}{|c|}{ Medio } & \multirow[b]{2}{*}{ Totales } \\
\hline & & El Mundo & El País & Servimedia & RTVE & \\
\hline \multirow{5}{*}{ Área } & Discapacidad & 0 & 9 & 85 & 9 & 103 \\
\hline & General & 8 & 8 & 9 & 43 & 68 \\
\hline & Modelo médico & 0 & 8 & 0 & 0 & 8 \\
\hline & Modelo social & 0 & 1 & 85 & 9 & 95 \\
\hline & Ninguno & 8 & 8 & 9 & 43 & 68 \\
\hline \multirow{2}{*}{$\begin{array}{l}\text { Valor } \\
\text { Morfosintáct } \\
\text { ico }\end{array}$} & Adjetivo & 5 & 4 & 3 & 3 & 15 \\
\hline & Nombre & 3 & 13 & 91 & 49 & 156 \\
\hline \multirow{2}{*}{$\begin{array}{c}\text { Campo } \\
\text { semántico }\end{array}$} & Estigmatización & 0 & 9 & 2 & 4 & 15 \\
\hline & No estigmatización & 8 & 8 & 92 & 48 & 156 \\
\hline \multirow{6}{*}{$\begin{array}{l}\text { Acción } \\
\text { afirmativa }\end{array}$} & Existe & 0 & 0 & 27 & 7 & 34 \\
\hline & No existe & 8 & 17 & 67 & 45 & 137 \\
\hline & Casos Ejemplares & 0 & 0 & 3 & 1 & 4 \\
\hline & Inclusión & 0 & 0 & 25 & 6 & 31 \\
\hline & Ninguno & 8 & 17 & 65 & 45 & 135 \\
\hline & Otro & 0 & 0 & 1 & 0 & 1 \\
\hline \multirow{4}{*}{$\begin{array}{c}\text { Valor } \\
\text { semántico }\end{array}$} & Discapacidad & 0 & 9 & 64 & 9 & 82 \\
\hline & Técnico & 2 & 1 & 13 & 15 & 31 \\
\hline & General & 6 & 3 & 4 & 24 & 37 \\
\hline & Desambiguación & 0 & 4 & 13 & 4 & 21 \\
\hline \multirow{2}{*}{ Omisión } & Se propicia & 0 & 0 & 3 & 2 & 5 \\
\hline & No se indica & 8 & 17 & 91 & 50 & 166 \\
\hline
\end{tabular}

Fuente: Elaboración propia

En el manual de estilo de RTVE aparece un epígrafe (5.13) "Discapacidad y Enfermedad" (RTVE: 85) dentro del tema "Cuestiones Sensibles" que dan cuenta de como este medio enfoca la discapacidad bajo el modelo médico. Por otra parte, se dice que "sólo se apuntará la discapacidad cuando sea razón de peso o gravedad en la información" (Ibid: 85) con lo que se impone una pauta de omisión, ya que se explicita que no se mencionará a menos que sea relevante con el fin de evitar, entendemos, el sensacionalismo. 
Paralelamente se muestra la preocupación por la representación de estereotipos o sesgos en la información y se insta a no definir a las personas por sus cualidades o carencias. Observamos en este sentido una diferenciación morfosintáctica al distinguirse entre "nombrar la enfermedad y no describir con ella a quienes la padecen" (Ibid: 85). Así, se insiste en mencionar la discapacidad mental como un complemento del nombre (que acompaña a persona, enfermo o individuo) y nunca como un adjetivo o nombre común, y se preserva la condición de humanidad del sujeto, i.e., "hablar de personas con síndrome Down y nunca de subnormales" (Ibid: 85).

Este capítulo presenta un párrafo, que consideramos ejemplo de acción afirmativa, dedicado a la integración. En él se hace partícipe y parte principal al periodista, de manera que "sus [de RTVE] profesionales están obligados a facilitar su integración y evitar cualquier forma de discriminación" (Ibid: 85). Otro ejemplo de esta proactividad es el texto del apartado 1.2.1.4, "Retransmisiones y programas deportivos", que enuncia que "los deportes practicados por personas con discapacidad tendrán la presencia adecuada; merecen atención especial y deben ser ofrecidos de manera atractiva como ejemplo de superación".

No obstante, los verbos y referencias asociados a la enfermedad, como padecer o afectar, son frecuentes.

La accesibilidad en el manual de RTVE es relativa al ámbito técnico y por tanto no al específico de la discapacidad (se asocia a términos como buscadores o webhosting). El adjetivo accesible se presenta vinculado al público infantil y al hecho de contexto de la persona que accede (desde cualquier lugar o momento). Este texto presenta como hemos visto por su frecuencia atención especial al acceso (Ibid: 11, 23, 24, 25 y 54; 44, 21 y 22), debido a que RTVE tiene un reglamento del "Derecho de Acceso".

Pasando al análisis del documento producido por Servimedia, la discapacidad surge en 70 ocasiones, lo que da cuenta de la importancia de esta cuestión en el manual. En él, se pueden leer reflexiones sobre la dependencia, el cuidado de los verbos que se asocian o la necesaria atribución de carácter de persona a quien presenta la discapacidad (Servimedia, 2006: 39-40). Este manual se centra en erradicar la estigmatización. Así, encontramos ejemplos como que no se es sordo, se es persona sorda (Ibid: 39) y se presenta una discapacidad como algo que no se padece o sufre (Ibid: 39-40). Las referencias a minusvalía y deficiencia insertas en el texto explican el cambio conceptual y se recomienda no utilizarlas (Ibid: 39, 42 y 40). El término diversidad funcional se mencionará "sólo en caso de que la fuente original la utilice literalmente" (Ibid: 40).

En el manual de estilo de Servimedia sí se atiende a la especificidad, puesto que se tratan diferentes tipos de discapacidad. De hecho, es el único de los textos en el que encontramos la conceptualización de la discapacidad visual, en contraste con la ceguera (Ibid: 42). 
Las nociones de accesibilidad aparecen ligadas a un campo semántico general o técnico (Ibid: 159, 162 y 32): se enlazan con la información digital, la diferenciación entre accesible y asequible y como principio general de redacción.

En el libro de estilo de EFE FUNDEU de 1995 (en el que dado el formato físico del documento no se han realizado análisis de frecuencias), se han encontrado las definiciones de 3 de los términos discapacitados (EFE, 1995: 179), minusvalía (Ibid: 214) y accesible que redirecciona a asequible (Ibid: 152). Con relación a la palabra discapacitados, el medio señala que preferirá incapacitados o minusválidos. Este texto recalca la diferencia entre minusvalía y minusvalidez, la segunda queda detallada como "calidad de minusválido", mientras la primera conserva las connotaciones de menor valía. Finalmente encontramos como en otros textos la desambiguación de los términos accesible y asequible, así una persona accesible es aquella de fácil trato y acceso.

En el manual de 2008 la conceptualización cambia y así, discapacitado (EFE, 2008: 228) puede usarse como "sinónimo de incapacitado, minusválido o disminuido", sin que se establezcan preferencias.

\section{Discusión}

Se han encontrado apariciones de los conceptos analizados en la mayoría de los libros de estilo. En general, el cambio de siglo e hitos como la Convención han puesto en desuso la minusvalía (invalidez y otros) a favor de la discapacidad. Por lo tanto creemos que nuestros resultados respaldan lo que Fernández-Cid apunta: "puede pensarse en la progresiva inclusión, por parte de los periodistas, de orientaciones y propuestas procedentes de ámbitos vinculados directamente a la discapacidad" (2010: 108).

Ejemplo de esta evolución se ve si comparamos la propuesta de El País, donde la minusvalía actúa como concepto superior para la discapacidad y deficiencia, frente a la propuesta de Servimedia (2006), que propone un cambio morfosintáctico en el uso de las palabras y que hace una mención expresa para no definir a la persona por una condición física o psíquica y en cambio hablar de persona con discapacidad. Ambas definiciones tienen una década de diferencia, la primera pertenece a los noventa del siglo XX y la segunda ya al siglo XXI.

La Convención identifica la vinculación de la discapacidad a la enfermedad y las actitudes asociadas culturalmente a problemática social como barreras. Estos dos factores pertenecen al modelo médico que se intenta superar. En consecuencia entendemos que los modelos propuestos en los manuales de Servimedia y RTVE, el segundo con matices, son los más próximos a las recomendaciones internacionales.

El primero, Servimedia, aboga directamente por la erradicación de las connotaciones semánticas y morfosintácticas, e incluye la extinción de los verbos que impliquen 
enfermedad o puedan ser negativos, como padecer o sufrir frente a presentar. RTVE, que apela al uso morfosintáctico correcto de la discapacidad, no obstante sigue describiendo la "Discapacidad y Enfermedad" dentro de "Cuestiones Sensibles" y utiliza habitualmente en sus descripciones verbos como padecer. No obstante, el texto al que remite es de 1980, década en la que está surgiendo el modelo social (Filkenstein, 1980; Stibbe, 2004). A su favor, este manual posiciona a los profesionales de la comunicación como actores en la tarea de integración social, un punto importante dentro de las recomendaciones de la Convención a fin de garantizar la participación plena y efectiva en igualdad de condiciones. No se olvida de la parte técnica ya que se insta a los trabajadores a usar los recursos oportunos para garantizar el acceso, aunque este concepto no se encuentra delimitado dentro del campo de la discapacidad sino que se presenta en un ámbito general de actuación. Asimismo, la Convención insiste en que los medios cuiden la imagen que se transmite de la discapacidad. En este sentido, RTVE, en su ámbito deportivo, intenta contar con el compromiso de los profesionales. Así, el deporte se convierte en estandarte con una cobertura adecuada y la presentación de perfiles ejemplares, un ejemplo claro de acción afirmativa.

El modelo médico-social (Filkenstein, 1980; Devlieger, 1999; Colenbrander, 2000; Stibbe, 2004; Observatorio Estatal de la Discapacidad, 2007; OMS, 2010; Ferreira, 2010), que conceptualiza a partir de los márgenes de normalidad y que delimita la deficiencia dentro del ámbito fisiológico, queda representado en El País. La OMS [Organización Mundial de la Salud] (2011: 5) a partir de la Convención propone un concepto más avanzado que hace énfasis en los factores personales y medioambientales y que se centra en la actividad, no en la deficiencia de la persona.

La accesibilidad desde los medios se asocia a la inteligibilidad y la comprensión (El Mundo, El País y Servimedia). Sin embargo no se mencionan la perceptibilidad, la interactividad y la robustez (Ribera, 2009) elementos por otra parte esenciales para la universalidad del acceso al contenido producido por los medios. En consecuencia en la accesibilidad no interactúa el diseño universal, ni se hace referencia a la normativa específica existente. Sin embargo esta es una de las herramientas principales para la eliminación de barreras para la comunidad de personas con discapacidad. La universalidad del acceso es la piedra angular de la revolución mediática actual gracias al avance de las tecnologías.

Ante la posibilidad de error entre las definiciones de asequible y accesible, la mayor parte de los textos exponen una desambiguación del término. Este hecho es un claro ejemplo de la relevancia de los libros de estilo como reguladores lingüísticos para los profesionales.

\section{Conclusiones}

Si bien se han descubierto aproximaciones a los principios y definiciones de la Convención y se observa una evolución en los conceptos desde los años ochenta, la 
conceptualización de la discapacidad visual propuesta por los organismos internacionales no ha terminado de cristalizar en los libros de estilo.

Se aprecian 3 estadios en los diferentes documentos estudiados que se asocian al momento de producción del material. Primero, se encuentra la unificación semántica a través de definiciones y recomendaciones de uso (El País, Servimedia, EFE). En segundo lugar, la utilización morfosintáctica, bien como adjetivo (la discapacidad define al individuo) (EFE) o como complemento del nombre persona que centra la importancia en la humanidad del individuo (Servimedia, RTVE). Por último, se cuidan los verbos asociados con el fin de eliminar las connotaciones asociadas a la discapacidad, de manera que se vetan términos como padecer o sufrir y se recomienda el uso de verbos neutros (Servimedia).

La asociación de la discapacidad al campo semántico de la enfermedad está culturalmente muy asentada y se reconoce ya por los organismos internacionales como una barrera actitudinal subyacente para la participación plena y efectiva. Se ha encontrado esta asociación una parte importante de los textos analizados.

Uno de los retos pendientes es el de la comprensión de la accesibilidad en el sentido que le da la Convención: asociada a la noción de diseño universal. En los libros de estilo esta idea aparece ligada a la inteligibilidad. Sin embargo, se soslayan la perceptibilidad, la interactividad y la robustez, incluidas también en el concepto de diseño para todos.

Se encuentra en estos textos algún ejemplo de acción afirmativa. Algunos soportes recomiendan el marco ideológico de perfiles ejemplares.

Parece hallarse una mayor preocupación en empresas relacionadas con la administración pública o el tercer sector, en contraposición con las empresas del sector privado.

\section{Bibliografía}

ALCOBA, S. (2009). Lengua, comunicación y libros de estilo. Barcelona. Disponible en: http://dfe.uab.es/dfeblog/salcoba/files/2009/07/libro-estilo.pdf [13-05-2013]

AUSLANDER, Gail K., \& GOLD, Nora (1999). "Disability terminology in the media: A comparison of newspaper reports in Canada and Israel", en Social Science \& Medicine, $\mathrm{n}^{\circ}$ 48, p.1395-1405.

COLENBRANDER, A. (2000): Disability Evaluation—Vision. Disponible en: http:// www.ski.org/Colenbrander/Images/Disability Eval Vis.pdf [15-05-2013]

DEVLIEGER, P. J. (1999). "From handicap to disability: language use and cultural meaning in the United States", Disability and rehabilitation, $\mathrm{n}^{\circ} 21$ (7), p. 346-354.

DOMÉNECH RIERA, X. (2010). "Historia de la Tiflotecnología en España”, No Solo Usabilidad, $\mathrm{n}^{\circ}$ 9. Disponible en: http://www.nosolousabilidad.com/articulos/ tiflotecnologia.htm. [15-05-2013] 
EFE (1995). Manual del Español Urgente. Catedra. Madrid. EFE (2008). Manual del Español Urgente. Catedra. Madrid.

AIMC (2013). Estudio General de Medios. Madrid Disponible en: http://www.aimc. es/spip.php?action=acceder_document\&arg $=2316 \&$ cle $=$ bb8ed3413dd68f0945dbef90411b9164cab1a5e8\&file=pdf\%2Fresumegm113.pdf [15-05-2013]

EL MUNDO (2002). Libro de estilo del diario El Mundo. Disponible en: http://www. masmenos.es/wp-content/uploads/2002/01/librodeestilo_elmundo.pdf [15-052013]

EL PAÍS (2002). Libro de estilo. El País 20 años. Ediciones El País. Disponible en: http://blogs.elpais.com/files/manual-de-estilo-de-el-pa\%C3\%ADs.pdf [15-052013]

FERNÁNDEZ- CID ENRÍQUEZ, M. (2010). "Mass Media, Image Conformation and Sense Construction Due to Disability", Politica y Sociedad, n 47 (1), p.105113

FERREIRA, M. A. V. (2010). "De la minus-valía a la diversidad funcional: un nuevo marco teórico-metodológico", Política y Sociedad, nº 47 (1), p. 45-65.

FILKENSTEIN, V. (1980). Attitudes and Disabled People: Issues for Discussion, New York: World Rehabilitation Fund.

GÓMEZ FONT, A. (2000). "Los libros de estilo de los medios de comunicación en español: necesidad de un acuerdo", Espéculo, Disponible en: http://pendientedemigracion.ucm.es/info/especulo/ele/g_font.html. [15-05-2013]

GÓMEZ VÁZQUEZ, M. (1998). "El Servicio de Documentación de El Mundo", Cuadernos de Documentación Multimedia. Disponible en: http://pendientedemigracion.ucm.es/info/multidoc/multidoc/revista/cuad6-7/gomez.htm [25-05-2013]

GÓMEZ, Y.; MARTÍN LLAGUNO, M.; FLÓREZ REVUELTA, F. (2011). "Medios de comunicación, discapacidad visual y tecnologías de la información y la comunicación. Estudio de la producción científica", Icono 14, nº 8(1), p. 408-422.

HERSH, M. A.; JOHNSON, M. A. (2007). Assistive Technology for Visually Impaired and Blind People, Springer. Disponible en: http://web.iitd.ac.in/ pvmrao/assistive book.pdf [25-05-2013]

JACKO, J. A., VITENSE, H. S. y SCOTT, I. U. (2003). "Perceptual impairments and computing technologies", en JACKO y SEARS: The human-computer interaction handbook: Fundamentals, evolving technologies and emerging applications (p. 504-522). Lawrence Erlbaum Associates. Mahwah, NJ.

OBSERVATORIO ESTATAL DE LA DISCAPACIDAD (2010). La discapacidad en España. Informe Olivenza 2010. Ministerio de Sanidad, Política Social e Igualdad. Disponible en: http://www.observatoriodeladiscapacidad.es/sites/default/ files/io/i_o_completo.pdf. [15-05-2013].

OMS (2011). World Report on Disability. Disponible en: http://whqlibdoc.who.int/ publications/2011/9789240685215_eng.pdf [15-05-2013]

ONU (2007). Convención de Derechos de las Personas con Discapacidad. Disponible en http://www.un.org/esa/socdev/enable/documents/tccconvs.pdf. [13-052013]. 
PEREIRA, O. (2009). Representações sociais dos atletas paralímpicos nos media impressos portugueses - estudo efectuado em dois jornais diários nacionais generalistas e dois jornais diários nacionais especializados em desporto. Tesis de Fin de Máster. Faculdade de Desporto. Universidade do Porto (Portugal) Disponible en http://repositorio-aberto.up.pt/handle/10216/13617. [11/9/2013].

PÉREZ TORNERO, J. M. (2009). "Televisión y libros de estilo. De la identidad normativa a la regulación comunitaria", en ALCOBA: Lengua, comunicación y libros de estilo. Premisas. Barcelona. p. 199-220

PIÑUEL RAIGADA, J. L. (2002). "Epistemología, metodología y técnicas del análisis de contenido", en Sociolinguistic Studies, n $\mathrm{n}^{\circ}$ 3(1), p.1-42.

RIBERA, M. (2009). "La nueva normativa de accesibilidad WCAG 2.0 y los documentos en Internet", Hipertext.net, ${ }^{\circ} 7$. Disponible en: http://www.upf.edu/hipertextnet/numero-7/wcag-2-0.html. [1-09-2013]

RTVE. Manual de Estilo de rtve. Directrices para los profesionales. Disponible en: http://manualdeestilo.rtve.es/ [21-05-2013]

SERVIMEDIA (2006). Periodismo social. El compromiso de la información. Libro de estilo. Servimedia. Madrid. Disponible en: http://www.servimedia.es/LibroEstilo.pdf. [23-05-2013]

STIBBE, A. (2004). "Disability, Gender and Power in Japanese Television Drama", Japan Forum, no 16 (1), p. 21-36.

\section{Notas}

1. El principio que insta a que el contenido deba ser "perceptible", es el primero y, hace referencia a su visibilidad a uno o más sentidos, ante lo cual es necesario el acceso a cualquier persona aunque esta sea por ejemplo una persona ciega o sorda (Ribera, 2009). Esto implica que es necesario traducir la información visual como las letras o las imágenes a información auditiva o táctil, o el contenido sonoro tiene que ser expuesto para que pueda ser percibido a través de la vista.

2. El principio "operable" exige que cualquier usuario debe ser capaz de realizar la interacción necesaria para actuar con el contenido (Ribera, 2009).

3. El último principio insiste en que el contenido sea "robusto", es decir, se encuentre "suficientemente descrito para poder ser leído con distintos lectores y con distintas tecnologías de asistencia ahora y en el futuro" (Ribera, 2009).

\section{Los autores}

Marta Martín Llaguno, Catedrática de Comunicación Audiovisual y Publicidad. Dra. en Comunicación Pública por la Universidad de Navarra es profesora de Deontología Publicitaria y de Teoría de la Comunicación en la titulación de Publicidad y RRPP. de la Universidad de Alicante. Autora de medio centenar de artículos indexados y de otros tantos capítulos de libros, ha participado en proyectos internacionales, europeos, nacionales y autonómicos, la mayoría de ellos centrados en salud, género 
y medios de comunicación. También ha dirigido equipos y proyectos de innovación educativa en comunicación. Directora del grupo de investigación COSOCO es miembro del grupo de investigación competitivo de la UA Salud Pública.

Andreu Castellet Homet es Doctor en Comunicación por la Universidad de Murcia (2012). Es profesor asociado en el área de Comunicación Audiovisual del Departamento de Información y Documentación de la Universidad de Murcia. El análisis de los mercados de contenidos digitales, particularmente los contenidos móviles, ha sido su principal ámbito de investigación, que desarrolla en del grupo e-Com de la citada Universidad. Ha creado el blog Pantalla Móvil, donde regularmente expone puntos de vista sobre las industrias digitales al hilo de la actualidad. Su experiencia de más de treinta años como periodista ha abarcado todo tipo de medios, y la información económica y financiera ha sido su especialidad principal.

Yordhana Gómez, doctoranda de la Universidad de Alicante, es Máster en Comunicación Social. Su principal campo de interés es la discapacidad visual y los conflictos para el acceso a los medios de comunicación de las personas con este tipo de discapacidad. Voluntaria de la ONCE (Organización Nacional de Ciegos Españoles), lleva más de 4 años trabajando por la autonomía y la integración. 